\title{
Lessons learned on the application of vibration absorbers for enhanced cannon stabilization
}

Eric Kathe

\author{
US Army Tank-Automotive and Armaments Command, \\ Armament Research, Development, and Engineering \\ Center, Benet Laboratories Watervliet Arsenal, NY \\ 12189-4050, USA
}

E-mail:ekathe@PICA.ARMY.MIL

This paper will summarize the successful application of muzzle-end vibration absorbers to reduce cannon vibration. This technology constitutes a weapons stabilization approach that focuses on passive mechanical structural modification of the cannon, rather than relying upon an external control law to actively cancel vibrations. Challenges encountered during field testing, non-ideal behavior, and performance evaluation using digital signal processing will be highlighted.

\section{Introduction}

Structural vibration of gun systems is becoming an increasingly important issue in the tank community due to increased exit velocity requirements of rounds used to defeat anticipated and current threat reactive armor. Increased velocity demands longer gun barrels and subsequently increased reacceptance to environmentally induced vibration. (Receptance is the ratio of deflection amplitude to force as a function of frequency [1].) Considering the strong desire to increase performance while the weapon platform is outmaneuvering its target, the need to introduce methods to attenuate the receptance of the gun system to environmental vibration becomes clear.

The increased system receptance has manifested itself in decreased weapon accuracy during live-fire bump-course testing of a developmental extended length 120-mm tank cannon (the XM291) mounted to a modified M1A1 tank conducted at Aberdeen Proving Ground, Maryland. The accuracy is particularly sensitive to the traversing speed of the tank over the bump-course, and increases for system configurations that have utilized a shorter barrel [2]. This degradation of accuracy is wholly absent for live-fire testing of the same gun system from a stationary mount [3] where the developmental system outperforms its infield counterpart, the 120-mm M256.

An effect relating to gun performance as well as accuracy is the yaw state of the bullet as it leaves the muzzle. In this context, yaw is the angle between the momentum vector and the axial centerline of the projectile. In general, barrel vibration activity that decreases accuracy also tends to increase the yaw state of the bullet at shot exit. Increased yaw at impact decreases penetrator performance [4]. This projectile condition is damped out during flight by the fins of the round, and their associated drag and range limitations. Thus, the effectiveness of the weapon for near-targets may be impaired, even though precision accuracy may not be a consideration under such circumstances.

For these reasons, the current focus of engineering efforts to enhance the accuracy and performance of extended length launchers is placed upon attenuation of the receptance of the gun system to environmentally induced barrel vibrations, while maintaining the length at levels that enable a muzzle velocity capable of defeating anticipated target armor. It is believed that this approach will achieve enhanced accuracy by reducing dispersion caused by variation in the initial conditions of the gun structure at the commencement of launch; i.e., shoot out of a straighter barrel.

Structural control of the barrel during the launch dynamics would prove a daunting task due to the particularly narrow time window during which corrective action must be taken - typically less than eight milliseconds. Coupled with the need for significant sensor information, high actuation power levels (due to the brief time window), and a severe operating environment; the application of smart structure technology to control launch dynamics is not viable for a developmental system that is targeted for near-term applications such as a 
potential M1A tank series gun upgrade. Such technology may however find applications for next generation systems.

For these reasons, intermediate technology solutions that are essentially compatible with the current M1A weapon platform and its subsystems have been sought.

\section{Traditional engineering solutions}

Several engineering approaches to reduce vibration receptance of gun systems have been applied, with differing advantages.

1) Stiffening the gun via thicker barrels raises density nearly in proportion to stiffness, especially near the muzzle end, where the cylinder is close to a thin-wall approximation.

2) Cradle/mount extension effectively decreases the cantilevered length of the gun, and has successfully increased modal frequencies [5]. However, extended cradles present significant weapon platform integration issues; e.g., they don't fit in the allotted space for a retrofit that does not require a new turret.

3) The application of composite overwraps to enhance the stiffness-to-density ratio has encountered challenges during firing, manifested as delamination of the composite from the gun steel. This is not to rule out the future of composite applications.

4) Active vibration suppression via the fire control and weapon stabilization system (the system that points the gun on target) has been considered. Although a promising candidate to enhance the performance, active control approaches are hampered by the performance limitations of the hydraulic elevation and azimuthal actuators, sparse sensory information, and integration with the existing control hardware and software.

\section{A muzzle end vibration absorber}

A muzzle end vibration absorber has been proposed as an intermediate technology enhancement that suffers virtually no integration issues with the current weapon platform [6]. Both a conceptual schematic and an image of the system utilized in the testing are shown in Fig. 1. Such an absorber concept was numerically modeled [7] and subsequently experimentally validated in a laboratory setting [8] prior to construction of the test fixture.

Details of the absorber integration with the gun system to enable function in a severe operating environment are available via a limited distribution report [9]. The primary function of the forward thermal shroud shown in Fig. 1 is to prevent thermal gradients within the barrel that would otherwise cause unacceptable distortions (e.g., direct sunlight heating one side of the barrel). The back of the forward shroud is affixed to the bore evacuator using a compression collar that enables a pivoting action; this prevents thermal distortions in the shroud from being transmitted to the barrel. The absorber fixture consisted of a simple spring collar mounted to the front of the shroud. This provides the spring action of the vibration absorber between the barrel's muzzle and the front of the forward shroud. Thus, the inertia of the shroud as it pivots was leveraged to provide mass for the vibration absorber [6]. This approach was termed the dynamically tuned shroud (DTS).

For pragmatic reasons, the free amplitude of the absorber relative to the barrel was severely restricted to a nominal value of plus or minus three millimeters. An energy absorbing rubber material was applied to the inner surface of the vibration absorber fixture to act as a snubber and prevent metal-to-metal impact.

The proof of principle fixture depicted utilized offthe-shelf helical springs to provide the elastic restoring force of the absorber. The springs were interchangeable with springs of differing stiffness to enable testing of various absorber parameters for optimizing overall system performance. Although shock absorbers could be integrated with the design to control damping levels, significant friction inherent in the mechanism prevented full control of this parameter. Thus the mechanism constituted a damped vibration absorber. Provisions were also included to add weights (not shown) to adjust the mass of the absorber.

A vibration absorber offers several clear advantages. The absorber constitutes a purely passive approach, and thus no sensor, actuator, or fire control integration is required. The absorber is fully compatible with all other engineering solutions. Further, the absorber does not interfere with the optical path used by the continuous muzzle reference sensor (CMRS). The principal disadvantage of a vibration absorber is that a moving part is applied to a particularly harsh dynamic environment albeit a simple moving part. 


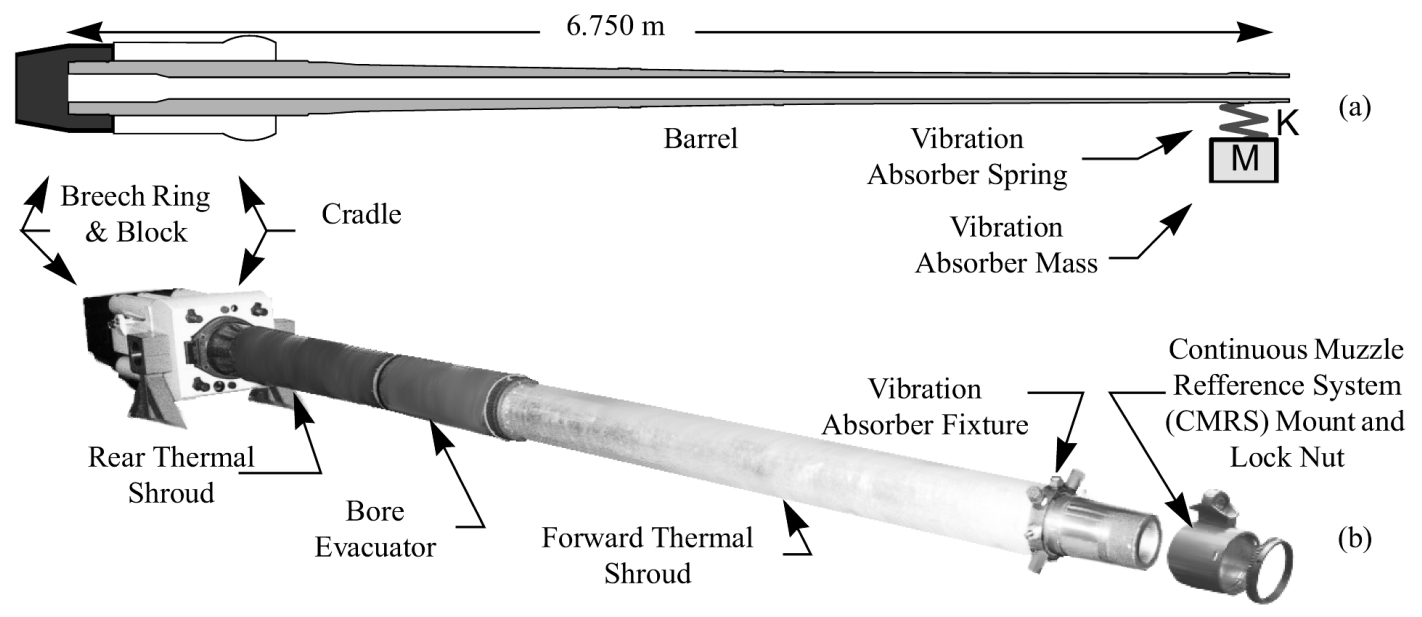

Fig. 1. Schematic depiction of a muzzle-end vibration absorber (a) and an image of the gun system tested (b).

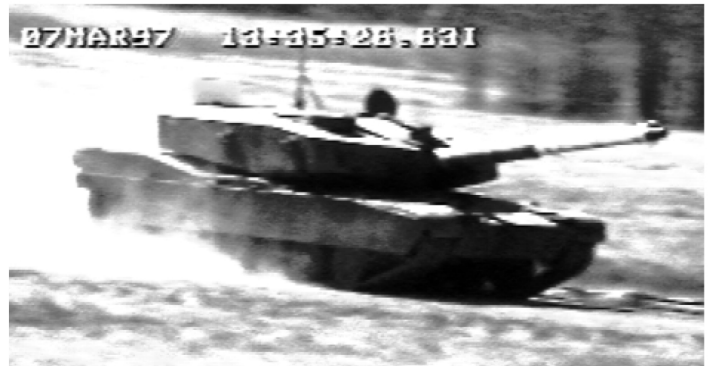

Fig. 2. Image of the modified M1A1 tank returning from a test.

\section{The tests}

The vibration absorber was mounted to a modified M1A1 tank depicted in Fig. 2. The tank modifications were extensive and included an XM291 120-mm gun system, and XM91 bustle autoloader, enhanced feed forward and feedback stabilization systems, a continuous muzzle reference system, extensive data collection, and telemetric data relay.

The XM291 gun system is $1.45 \mathrm{~m}$ longer than the current $5.3 \mathrm{~m}$ long M256. Simply put, this additional length provides greater working volume for launch. This results in greater muzzle velocities with lower propellant temperatures and pressures than would be required by an M256. Because the XM291 is longer, it is more susceptible to flexural vibrations.

Testing was conducted on the RRC-9 stabilization course at Aberdeen Proving Ground, Maryland. The course consists of a gravel road with several dozen steel ramp bumps spaced along its length. The bumps are all introduced in pairs, one for each track of the tank, such that only pitch and heave (not roll) is introduced by the bumps.
Two types of bumps were used. Type A bumps consist of a six-inch $(0.15 \mathrm{~m})$ rise over a six-foot $(1.8 \mathrm{~m})$ span, and type B consist of a three-inch $(0.075 \mathrm{~m})$ rise over two feet $(0.6 \mathrm{~m})$. The geometry of the bumps and the time sequence at which they are encountered at fifteen miles per hour $(6.7 \mathrm{~m} / \mathrm{s})$ are shown in Fig. 3 .

The gun stabilization system was maintained in the active mode during the testing. This control system maintains the gun-pointing angle (as seen at the turret and trunnions) fixed relative to earth in both elevation and azimuth. Aside from live-fire testing, this is the most realistic test available to assess the performance of the gun barrel vibration absorber.

Three runs were made down the bump-course for both the base-line and absorber modified gun systems. A total of three absorber configurations were tested. The effective spring rates for the vibration absorber collar were $23 \mathrm{kN} / \mathrm{m}, 48 \mathrm{kN} / \mathrm{m}$, and $24 \mathrm{kN} / \mathrm{m}$ for configurations $\mathrm{A}, \mathrm{B}$, and $\mathrm{C}$, respectively. All three used the inertia of the forward thermal shroud as shown in Fig. 1. The mass of the shroud is $30 \mathrm{Kg}$ distributed nearly evenly along its length of $2.2 \mathrm{~m}$. Configuration $\mathrm{C}$ included an additional $9 \mathrm{Kg}$ mass at the front collar of the forward thermal shroud. For the base-line testing, the vibration absorber mechanism was removed and the remainder of the system was restored to its original condition [9].

\section{The analysis}

\subsection{Data collected}

Data recorded during the testing included a measure of vertical barrel flexure, horizontal barrel flexure, vertical pointing error, and horizontal pointing error. 


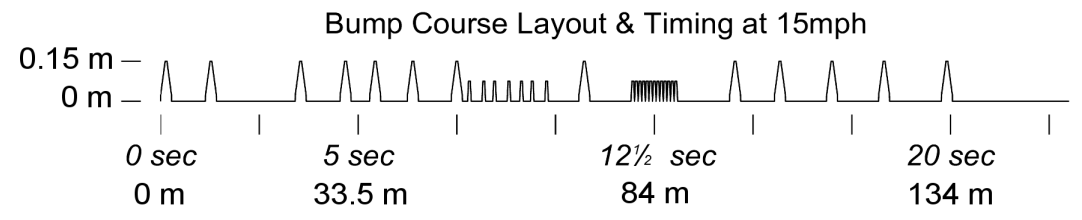

Fig. 3. Exaggerated plot of bump-course profile and corresponding time axis for a fifteen MPH course run.

The flexure is a measure of the difference between the pointing angle of the gun at the cradle and the pointing angle of the gun at the muzzle. The vertical and azimuthal mount angles are measured via resolvers at the trunnions and turret ring, respectively; while the muzzle angles are measured optically by a system (the CMRS) that bounces a source beam from the turret off of a mirror mounted to the muzzle of the barrel and measures the deflection of the returned beam. The deflection of the returned beam correlates to the angular deflection of the muzzle.

The pointing error is a measure of the difference between the command pointing direction and the direction measured by the aforementioned resolvers at the cradle. With the stabilization in the active mode, the command pointing direction is fixed relative to earth. The semantics are a bit awkward as the pointing direction of a non-straight barrel is somewhat of a misnomer and is not likely best represented by the direction measured at the cradle alone.

To ensure that only consistent data was used in the analysis, a method identifying the bump-course - in the measurement data - had to be developed. This need was caused by a lack of consistency in the start and end times of the data records.

The series of thirteen type B bumps encountered at $84 \mathrm{~m}(121 / 2$ seconds bump-course time) was found to provide a distinct signature in the horizontal bending data near the ten-second location in the data files, indicating truncation of the first seconds of the data. This signature point was quantitatively identified using a narrow band-pass filter constructed and implemented in MATLAB (The Mathworks, Natick, MA) to identify the peak that is asserted to occur at $121 / 2$ seconds bump-course time. The data sets were then truncated to begin at three seconds and end at twenty-two seconds bump-course time to provide a consistent data set for further analysis [9].

This approach does not make-up for uncontrollable variation in speed and an occasionally straddled bump while traversing the course. This testing is particularly challenging to the driver when the bumps are encountered in a series; the reaction of the turbine power-plant is not instantaneous and the drag of the bumps is significant. Therefore direct correlation analysis, bumpfor-bump is difficult.

\subsection{Root-mean-square (RMS) amplitude}

All of the data has been normalized by the RMS amplitude of the vertical bending angle. This value would provide specific accuracy information on the gun system. This RMS flexure is on the order of one milliradian, representing an error magnitude that correlates to a target with a radius of one meter at a distance of one kilometer. The normalized RMS values are listed in Table 1.

Clearly, the configuration of vibration absorber C, was the best overall performer. Configurations A and B appear to have shifted some energy from vertical bending into horizontal bending, although all three reduced vertical bending. The absorbers had little affect on the pointing error RMS values.

\subsection{Power spectra}

The power spectra for the base-line and vibration absorber $\mathrm{C}$ were computed using a 1024 element Hanning window (2.2 seconds at the data sampling frequency of $463 \mathrm{~Hz}$ ). An overlap of half the window size was used to reduce leakage effects from the non-stationary behavior of the bump-course. The power spectra for each of the three runs were subsequently averaged to reduce sensitivity to variation in traversing the bumpcourse. The results are displayed in Fig. 4.

\section{Discussion}

The vibrations of the gun system can roughly be broken down into three frequency regimes: 
Table 1

Normalized RMS performance of the configurations tested.

\begin{tabular}{lcccc}
\hline Configuration & Vertical bending & Horizontal bending & Vertical pointing error & Horizontal pointing error \\
\hline A & $94.3 \%$ & $61.4 \%$ & $44.3 \%$ & $36.5 \%$ \\
B & $82.8 \%$ & $52.2 \%$ & $4.37 \%$ & $41.2 \%$ \\
C & $76.2 \%$ & $43.4 \%$ & $39.3 \%$ & $35.9 \%$ \\
Base-line & $100 \%$ & $47.8 \%$ & $41.0 \%$ & $37.6 \%$ \\
\hline
\end{tabular}

\subsection{Forced vibration near $2 \mathrm{~Hz}$}

Although the entire tank is one structure, the hull upon its suspension may be considered a forced excitation platform that provides force input into the gun and mount structure. The fundamental modes of the tank are low (around $1 \mathrm{~Hz}$ ) due to its need for a compliant suspension to traverse rough terrain. Vibration energy resulting from traversing the bump-course is passed through the tank suspension (which acts as low-pass filter for frequencies above its fundamental modes) to the gun system.

The hydraulic stabilization system has been designed to control the pointing error as measured at the cradle. The performance of this feedback control system is limited by the finite actuation response available. The disturbance and control energy in this low frequency range result in a forced vertical bending mode response, despite the lack of receptance of the gun to this low frequency excitation.

\subsection{Fundamental horizontal mode near $7 \mathrm{~Hz}$}

In the horizontal orientation, the barrel is coupled to the relatively massive turret by the two horizontally opposed trunnion pins. These pins may be considered to mimic a very stiff torsional spring that couples the azimuthal angle of the barrel -at the trunnions- to the turret. In addition, the trunnions prevent linear deflection relative to the turret in the horizontal plane. Thus the trunnions provide two nearly rigid constraints to the barrel. Since the mass and rotational inertia of the turret are much higher than that of the gun system, the boundary condition may be expected to approach that of a cantilevered barrel in flexure, with a rigid body mode that is not discernible in the flexure data.

The first two fundamental modes of a nearly rigid boundary condition have been approximated for the gun system using beam finite elements. The modes have been computed to be $7.24 \mathrm{~Hz}$ and 25.0 [10]. Instrumented impact testing of the structure in a controlled laboratory setting has demonstrated the modes to be 7.25 Hz and 25.4 Hz [8]. This first cantilevered mode compares favorably with the horizontal bending power spectra in Fig. 4.

The cause of the notch in the center of the power spectra for this mode is unknown. Its existence for both the absorber and base-line cases precludes it from being a consequence of the absorber itself. (This notch was absent for absorber configurations A and B and was replaced with a peak at the notch with a power spectrum amplitude nearly three times higher [9]. This is where the horizontal RMS energy in Table 1 is located.)

To better illustrate the disparity in size between the gun system and turret, a computer-aided design image of the turret and barrel structure is depicted in Fig. 5.

\subsection{Fundamental vertical mode near $19 \mathrm{~Hz}$}

In the vertical orientation, the barrel is coupled to the turret by the two trunnion-bearings, which provide for pivoting action. In addition, the elevation mechanism provides control actuation some distance behind the trunnions (about a half of a meter). The trunnions prevent vertical translation of the gun system relative to the turret and hull with a nearly rigid constraint, and little torsional resistance. The cantilevered configuration of the barrel is maintained by the vertical force input of the elevation system. Operated by hydraulics, and implemented with a control scheme, the constraint of the elevation system is not nearly rigid, but rather much softer.

This forgiving servo-compliance is essential to prevent the force input from the suspension from reeking havoc with the stabilization and gun systems; it is desirable to decouple the gun system from the pitch and heave of the tank.

The center of mass of the gun system is not coincident with the trunnions but rather over hangs them by some distance. An open-loop control term, based upon a vertically oriented accelerometer fixed to the turret near the trunnions is used to compensate in real time for the moment created by the inertia of the gun at the center of mass and the acceleration at the trunnions. This enables the soft feedback servo-control parameters for the imbalanced system. 


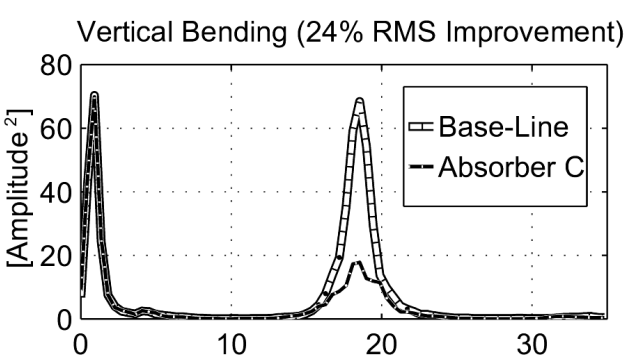

Horizontal Bending (9\% RMS Improvement)

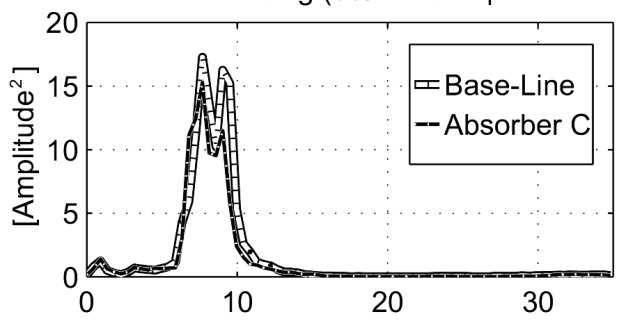

Vertical Pointing Error (4\% RMS Improvement)

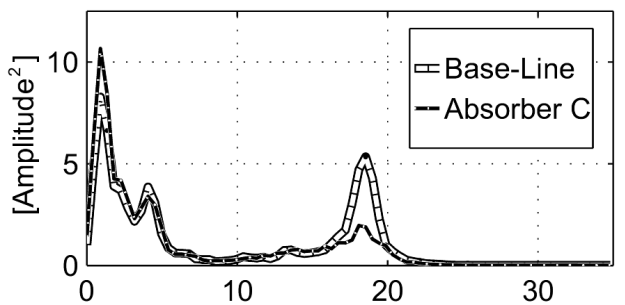

Horizontal Pointing Error (4\% RMS Improvement)

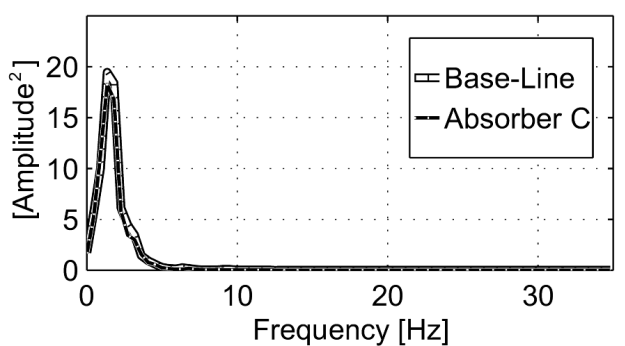

Fig. 4. Juxtaposed power spectra.

The first two flexible modes of a pinned free boundary condition have been evaluated using beam finite elements for the gun system. The resulting modes were computed to be $19.1 \mathrm{~Hz}$ and $32.4 \mathrm{~Hz}$ [10]. The first pinned-free mode compares favorably with the vertical bending power spectra in Fig. 4. An illustration of the vertical system is depicted in analogy with Fig. 5 in Fig. 6.

The cause of the large amount of vibration energy that is exciting the first vertical mode of the gun system

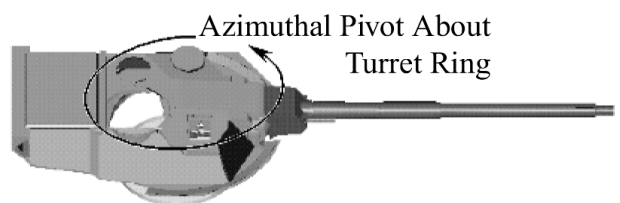

Fig. 5. Image of the coupled barrel and turret structure that vibrates horizontally with an open-loop rotational rigid body mode about the turret ring.

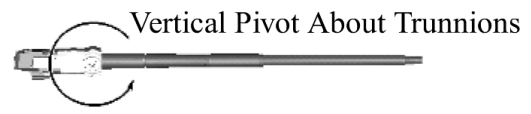

Fig. 6. Image of the barrel structure that vibrates vertically with an open-loop rotational rigid body mode.

is not clear. The second horizontal mode of the gun system is near in frequency, yet nearly no discernible energy is in its power spectrum above twelve $\mathrm{Hz}$. At well over sixty tons in mass, the tank is a very effective low-pass filter and should drop off nominally at twenty decibels per decade from $1 \mathrm{~Hz}$ fundamental, as would any other second-order damped system.

It has been suggested by Dr. Mark Kregal, ARL, that in this frequency range, the turret exhibits vibration as a rigid body in a rocking motion relative to the hull whose pivot is orthogonal to the azimuthal axis. This could be the result of clearances caused by out-of-plane imperfections between the mating surfaces of the hull and turret that bear the weight of the turret - as the tank traverses rough terrain, the turret may rattle. Such a structural coupling could cause a migration of this vibration energy to the gun system [11].

Another candidate for the high frequency source would be the open-loop control term used to counter the overhanging center of mass of the gun system. This term, although effective at maintaining the rigid body mode on target, applies a predictable disturbance bending moment to the gun system and vertical load to the turret and hull through the non-compliant trunnion bearings. Thus there is the potential for an undesirable feed-back loop, although the disparity in mass between the tank and gun system should lessen this type of effect.

A higher order open-loop control term could counter any negative effects on performance by leveraging the inverse dynamics of a gun system model to compensate for the predictable disturbance applied by the current open-loop term. This would also provide enhancement to the low frequency disturbance, regardless of its validity as a cause for the high frequency disturbance. 


\subsection{Performance in vertical bending}

The absorber performance was most pronounced in vertical bending, currently believed to be the largest contributor to degraded performance. The attenuation of the vertical bending was achieved without the migration of energy to other portions of the vertical bending spectrum. However, the absorber had little affect on the low frequency response that is asserted to be a direct result of the tank suspension.

This lack of low frequency performance is to be expected. A low frequency absorber would require either excessive mass to lower its natural frequency or an excessive amplitude envelope due to the increased displacements that would result if softer springs were used to lower its frequency.

\subsection{Performance in horizontal bending}

Of the three absorber configurations analyzed, two made horizontal bending significantly worse. The cause of the increase in horizontal bending for these two cases has been postulated to be the migration of vertical mode energy into the horizontal mode. The reason for the absence of this effect for absorber configuration $\mathrm{C}$ is not clear.

The shifting of this energy may be due to the severe amplitude restrictions placed upon the vibration absorber. Snubber engagement is a common event, and it is likely to occur with the most momentum in the vertical orientation. The momentum transfer will generally occur off the vertical axis with a significant vector component delivered into the horizontal mode. For example, the vibration absorber ring may impact the muzzle with vertical momentum, but strike the muzzle at a two o'clock position and thus rebound up and right, while forcing the barrel down and to the left.

To attempt to counter the migration of energy into the horizontal mode, future efforts will tune the horizontal and vertical mode absorbers separately via orientation dependent spring constants. An alternative would be to constrain the absorber from relative motion in the horizontal mode.

Regardless, modest increases in horizontal bending energy are worth significant reductions in vertical bending energy. This is true as the geometric error (Euclidean norm) is dominated by the larger vertical error. It is also valid, because most targets tend to be much wider than they are tall.

\subsection{Performance in vertical pointing error}

The effect of the vibration absorber on vertical pointing error was a zero-sum-gain in the time domain. As can be seen in Fig. 4, the energy was shifted from the $19 \mathrm{~Hz}$ mode to much lower frequencies, a trait shared by the two other absorbers configurations [9]. This provides significant advantage for any active control scheme using the existing hardware.

\subsection{Performance in horizontal pointing error}

The effect of the absorber on horizontal error was negligible. This lack of horizontal pointing sensitivity is likely caused by the large inertia of the turret structure that must be overcome. (Recall that in the vertical mode, only the gun is pointed. In the horizontal mode, the entire turret is pointed.) This error signal is not likely to be reduced by a simple lightweight passive device, but neither is it greatly exacerbated by longer barrels.

\section{General observations}

It became clear during the testing that snubber engagement was a common occurrence. The engagement of the snubber resulted in a tendency for the shroud to rotate about its centerline during testing. This was particularly frustrating as the top springs of the absorber spring collar were preloaded in compression to hold the static weight of the shroud above the barrel. As the shroud would rotate, the preload and weight would conspire to hold the shroud against the barrel. An effective field repair was to wrap the pivoting connection between the back of the forward shroud and the compression collar that held it against the bore evacuator with a single hoop wrap of duct tape. The repair offered little resistance to the intended low angle pivoting of the shroud while it prevented rotation.

The common occurrence of snubber engagement would suggest that the effectiveness of the vibration absorber was due largely to an impact absorber behavior. Impact absorbers, such as the lead shot used in a dead-blow hammer, use inelastic collisions to reduce energy. Credence to this effect may be gained by noting it was the absorber with the largest mass that had the greatest effect, despite the fact that its natural frequency was the farthest from the vertical bending mode that it attenuated so well. 

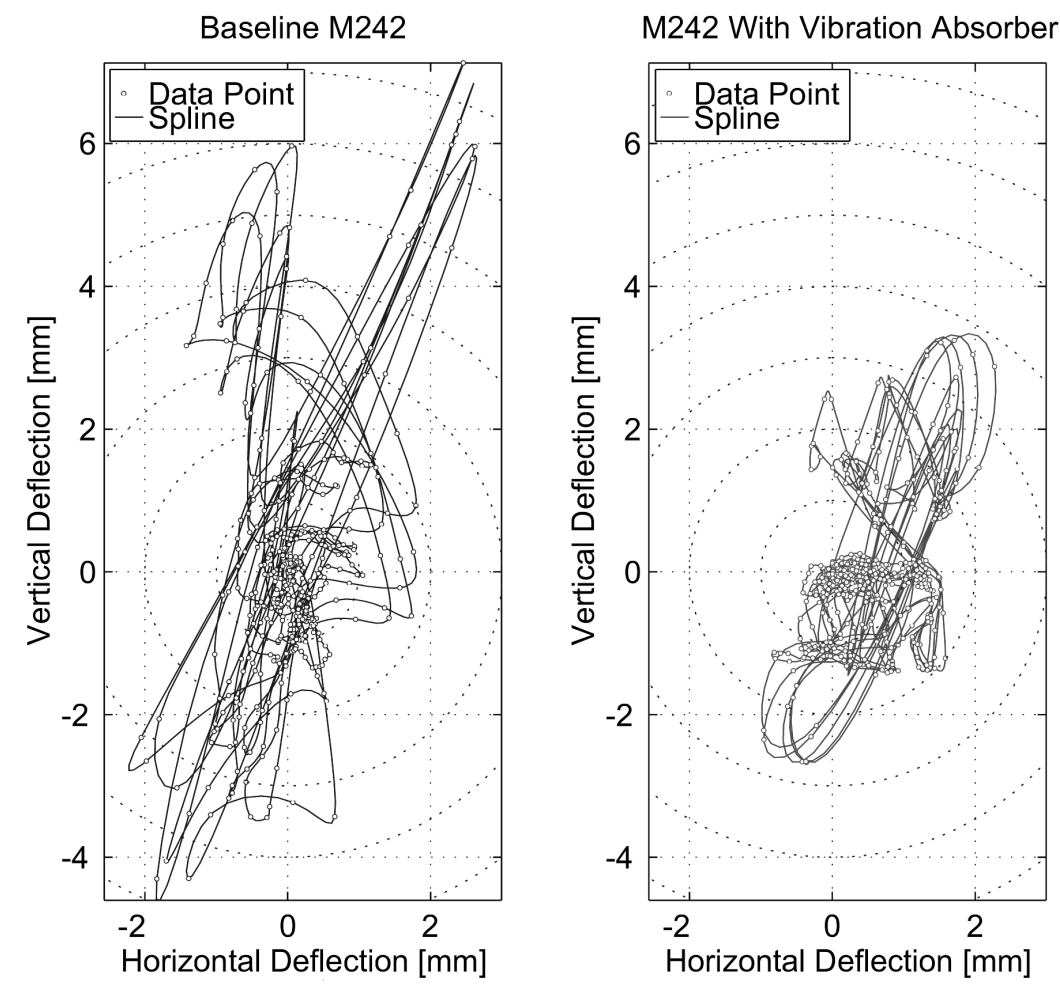

Fig. 7. Orbit plots from five round fire of a test $25 \mathrm{~mm} \mathrm{M} 242$ gun at Benét.

\section{Future work / preliminary results}

Although this work concentrated on the design of a vibration absorber to reduce vibrations caused by external excitation, the concept may also be applied to attenuate barrel flexure caused by successive fire. Preliminary results of such a test firing are depicted in Fig. 7. The tests demonstrated that the vibration amplitude could be essentially cut in half using a simple vibration absorber. Evaluation of the effect on accuracy was not possible due to the short trajectory of the projectile flight within the Benét Gun Dynamics Laboratory.

\section{Conclusions}

This testing set out to demonstrate the proof-ofprinciple that a simple muzzle end vibration absorber could significantly enhance the structural dynamics of a tank cannon. This has been demonstrated for absorber configuration C, as shown in Table 1 and Fig. 4. Therefore, investment in a rugged fixture to endure a firing environment is warranted.
It must be emphasized that the performance gain demonstrated was achieved with virtually no impact on any other portion of the weapon system and no restrictions placed upon other future enhancement options.

Little direct correlation between the flexural state of the barrel and the ultimate impact on accuracy and performance is available. However, much indirect evidence indicates that shooting out of a vibrating barrel is not as effective as shooting out of a quiet barrel. Strong evidence for static curvature effects (manufacturing tolerance, thermal gradients, and gravity droop) on accuracy has been published. (See for example Wilkerson [12], Bundy [13], and Kathe et al. [14].)

\section{Acknowledgments}

The author would like to acknowledge the support of Roger Billington and Charles Cording of the Program Manager - Tank Main Armament Systems, (PM-TMAS), Picatinny Arsenal, New Jersey; technical collaboration with Drs. Steven Wilkerson and Mark Kregal, Army Research Lab, (ARL) and Peter McCall, Army Test Center, (ATC), Aberdeen Proving Grounds, Maryland, and Professor Andrew Lemnios, Rensselaer 
Polytechnic Institute, Troy, NY; and the assistance of Michael Soja, Albert Pflegl, and dedication of Edmund Vanderwerken, of Benét Laboratories.

\section{References}

[1] D.J. Ewins, Modal Testing: Theory and Practice, Wiley, New York, 1984.

[2] P. McCall, ATAC TEST RESULTS: Summary of Test Configuration and Test Results from 1993 to Present, Presented at a Meeting of the Program Manager - Tank Main Armament Systems (PM-TMAS), Picatinny Arsenal, NJ, 22 March 1996.

[3] M. Soja, XM291 Hardstand Fire Test Results, presented at Joint Meeting of PM-ABRAMS, PM-TMAS, and Benet Laboratories, Watervliet Arsenal, NY, 06 December 1995.

[4] E.M. Schmidt and W.F. Donovan, A Technique for Reduction of Launch-Induced Perturbations, in: Proceedings of the Eighth US Army Symposium on Gun Dynamics, G. Albert Pflegl, ed., ARDEC Technical Report ARCCB-SP-96032, Benet Laboratories, Watervliet, NY, May 1996, pp. 12.1-7.

[5] R.G. Gast et al., Accuracy Enhancement of the 120-mm XM291 Gun, in: Proceedings of the Eighth US Army Symposium on Gun Dynamics, G. Albert Pflegl, ed., ARDEC Technical Report ARCCB-SP-96032, Benet Laboratories, Watervliet, NY, May 1996, pp. 22.1-28.

[6] E. Kathe, Gun Barrel Vibration Absorber, US Patent 6167794 , January 2001

[7] E.L. Kathe, Design of Passive Vibration Absorber to Reduce Terrain-Induced Gun Barrel Vibration in the Frequency Do- main, in: Proceedings of the Eighth US Army Symposium on Gun Dynamics, G. Albert Pflegl, ed., ARDEC Technical Report ARCCB-SP-96032, Benet Laboratories, Watervliet, NY, May 1996, pp. 28.1-17.

[8] E.L. Kathe, Design and Validation of a Gun Barrel Vibration Absorber, Proceedings of the 67th Shock and Vibration Symposium: Volume I, Published by the Shock and Vibration Information Analysis Center (SAVIAC), Monterey, CA, 18-22 November 1996, pp. 447-456.

[9] E.L. Kathe, Performance Assessment of a Synergistic Gun Barrel Vibration Absorber During Bump-Course Testing, ARDEC Technical Report ARCCB-TR-97022, Benet Laboratories, Watervliet, NY, September 1997.

[10] E.L. Kathe, MATLAB Modeling of Non-Uniform Beams Using the Finite Element Method for Dynamics Design and Analysis, ARDEC Technical Report ARCCB-TR-96010, Benet Laboratories, Watervliet, NY, April 1996.

[11] M. Kregal, Private Communication, July 1997.

[12] S. Wilkerson, The Effect of Initial and Gun Mount Conditions on the Accuracy of Kinetic Energy (KE) Projectiles, US Army Research Laboratory Technical Report ARL-TR-895, Aberdeen Proving Ground, MD, November 1995.

[13] M.L. Bundy, Thermally Controlled Bore Straightness During Firing, in: Proceedings of the Eighth US Army Symposium on Gun Dynamics, G. Albert Pflegl, ed., ARDEC Technical Report ARCCB-SP-96032, Benet Laboratories, Watervliet, NY, May 1996, pp. 6.1-12.

[14] E. Kathe, S. Wilkerson, A. Zielinski and A. Baz, Gun Structural Dynamic Considerations for Near-Target Performance of Hypervelocity Launchers, US Army Research Laboratory Technical Report ARL-TR-1795, Aberdeen Proving Ground, MD, September 1998. 

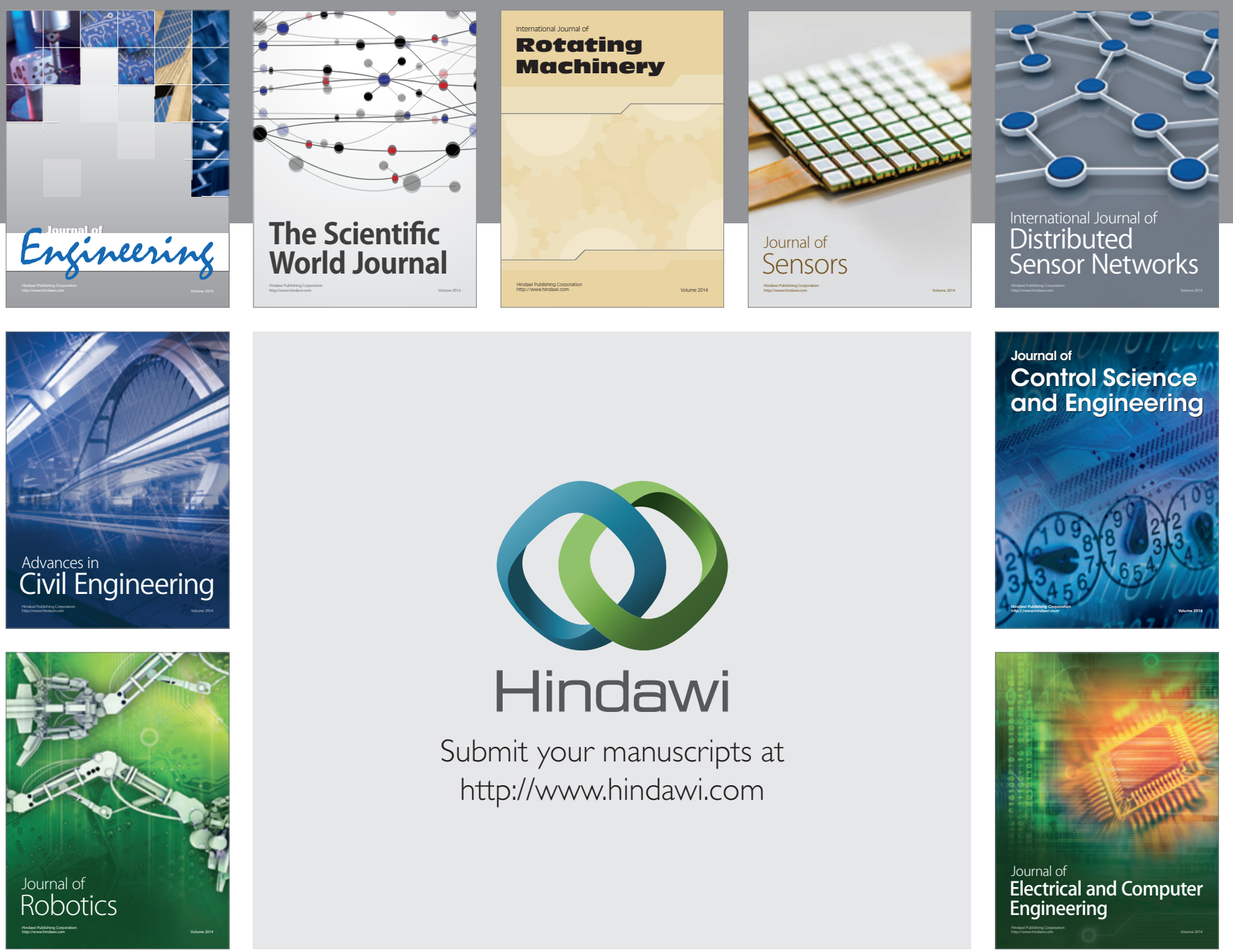

Submit your manuscripts at

http://www.hindawi.com
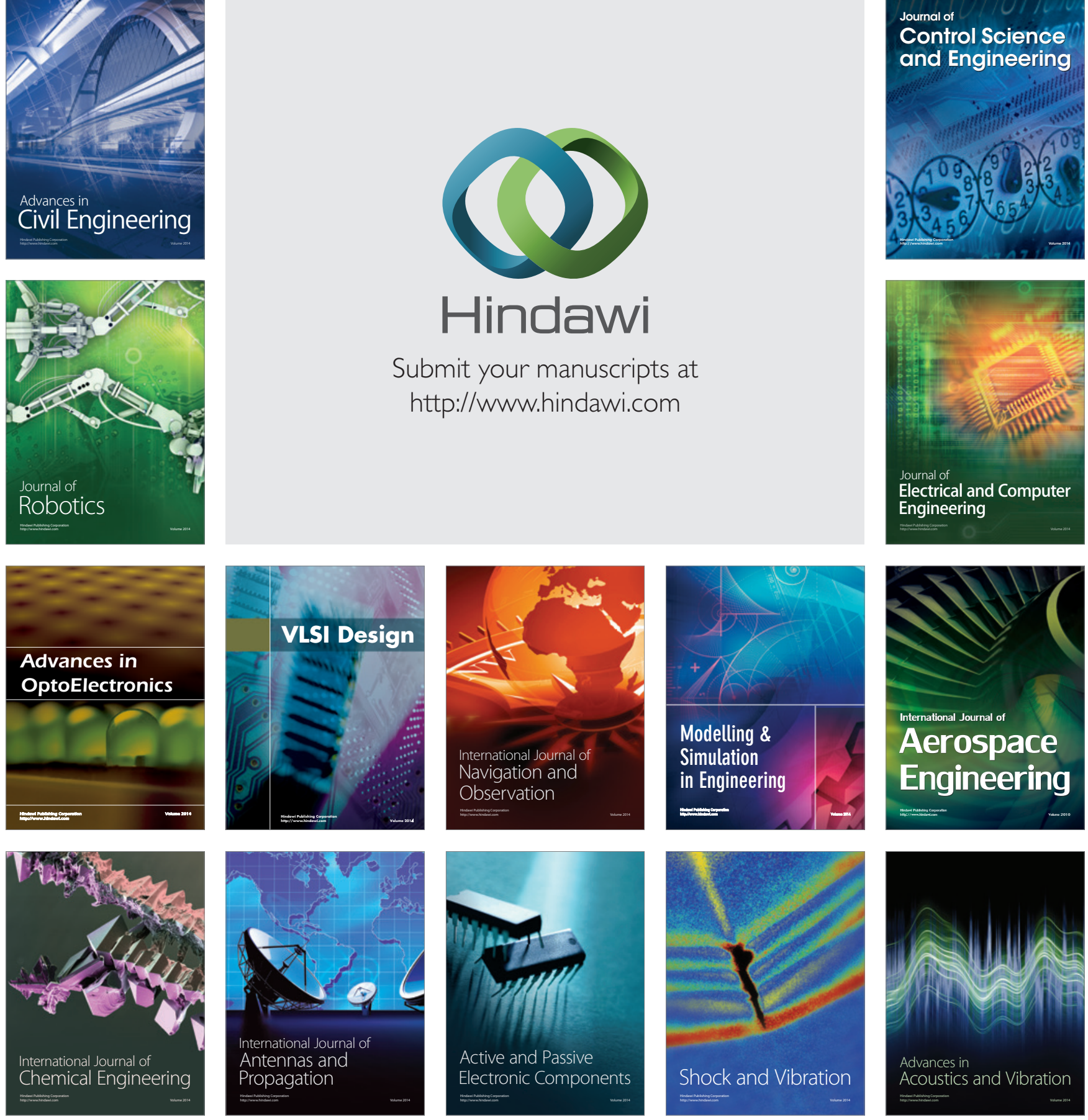